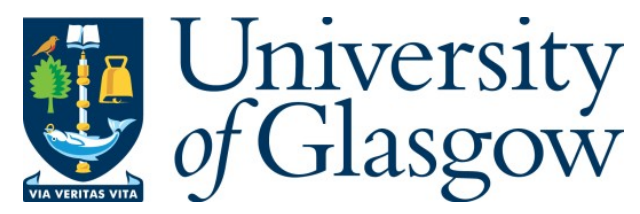

Ge, Y., Zhang, Y., Booth, J., Weaver, J., and Dobson, P. (2016) Quantification of probe-sample interactions of a scanning thermal microscope using a nanofabricated calibration sample having programmable size. Nanotechnology, 27(32), 325503.

There may be differences between this version and the published version. You are advised to consult the publisher's version if you wish to cite from it.

http://eprints.gla.ac.uk/120317/

Deposited on: 06 July 2016

Enlighten - Research publications by members of the University of Glasgow http://eprints.gla.ac.uk 


\title{
Quantification of probe-sample interactions of a scanning thermal microscope using a nanofabricated calibration sample having programmable size
}

\author{
Yunfei Ge ${ }^{1}$, Yuan Zhang $^{1}$, Jamie A. Booth ${ }^{2}$, Jonathan M. R. Weaver ${ }^{1}$, and \\ Phillip S. Dobson ${ }^{1}$ \\ ${ }^{1}$ School of Engineering, University of Glasgow, Rankine Building, Oakfield Avenue, \\ Glasgow G12 8LT, United Kingdom \\ ${ }^{2}$ Mechanical Engineering Department, University of California, Santa Barbara CA \\ 93106, USA \\ E-mail: y.ge.1@research.gla.ac.uk \\ Phil.Dobson@glasgow.ac.uk
}

May 2016

\begin{abstract}
We report a method for quantifying scanning thermal microscopy (SThM) probe-sample thermal interactions in-air using a novel temperature calibration device. This new device has been designed, fabricated and characterized using SThM to provide an accurate and spatially variable temperature distribution that can be used as a temperature reference due to its unique design. The device was characterized by means of a microfabricated SThM probe operating in passive mode. This data was interpreted using a heat transfer model, built to describe the thermal interactions during a SThM thermal scan. This permitted the thermal contact resistance between the SThM tip and the device to be determined as $8.33 \times 10^{5} \mathrm{~K} / \mathrm{W}$. It also permitted the probe-sample contact radius to be clarified as being the same size as the probe's tip radius of curvature. Finally, the data was used in the construction of a lumped-system steady state model for the SThM probe and its potential applications were addressed.
\end{abstract}




\section{Introduction}

In recent years scanning thermal microscopy (SThM) has become a widely used tool for the investigation of sub-micron heat transfer [1][2][3]. It has been applied to the study and development of nanostructured materials, such as superlattices [4][5], and micro/nano electronic devices, for example, nanowires [6][7].

SThM promises to be a powerful tool for obtaining sample temperature at the nanoscale due to its high spatial resolution when working in "passive mode" (temperature measurement with minimal self-heating) [8]. However, one difficulty when using SThM is that the temperature of its tip can only be acquired indirectly as an electrical signal [9][10]. This is compounded by the fact that, in passive mode, the probe temperature is always lower than that of the sample as a result of the thermal resistance of the tip-sample contact [1][11][12]. Therefore, for quantitative measurement, temperature calibration, which associates a well-known sample temperature with the electrical signal of the probe, is essential. To date, several methods have been developed to calibrate SThM probes following on from the Peltier module first used to calibrate thermocouple probes by Luo et al. [11]. Although some of these approaches are relatively advanced, for example, the 4-terminal-resistor calibration device developed by Shi et al. [13], the resistance heater embedded in a $\operatorname{SiN}_{\mathrm{x}}$ membrane [14], and the Johnson noise thermometer on a membrane [15][16], they all suffer from poor temperature uniformity which impedes their further application.

Another facet of calibration is the quantification of SThM probe-sample thermal interactions. When working in "active mode" (probe is both a heater and a thermometer), SThM can be used to explore the thermal properties of materials, for example nanowires[17][18], nanotubes [19] and graphene [20][21][22]. In order to make these measurements quantitative, it is important to understand probe thermal interactions at the nanoscale. This is complicated by the fact that many SThM probe structural dimensions are of comparable size to the mean free path (MFP) of heat carriers. Several studies have interrogated the SThM tip-sample contact in order to eliminate the ambiguity of the thermal contact area [3][23] and estimate the thermal resistance associated with the tip-sample constriction [3][24][25][26][27][28][29]. However, these works are either based on the usage of thermocouple probes with a thermal sensor localized at the end of the tip, or have been carried out under vacuum conditions. Furthermore, they have failed to reach agreement on the thermal contact conductance (or resistance) proposing values that range from tens to hundreds of $n \mathrm{~W} / \mathrm{K}[25,26,30-32]$. Recently, several groups have shown that the DMT (Derjaguin, Muller, and Toporov) model [33] is invalid for analyzing tip-sample nanoscale contact 
as it fails to match experimental data [3][23][34]. As a result, recent studies tend to regard the probe's tip radius of curvature $(\sim 50 \mathrm{~nm}$ for the probes in this work [35]) as the real contact radius. However, this has not been confirmed for a large range of conditions and probes. In particular, measurements under ambient conditions using probes with a nonlocalized thermal sensor, in particular the $\mathrm{SiN}_{\mathrm{x}}$ probe, are still needed. As a consequence, unlike the doped silicon and Wollaston probes for which thermal models have been well-established [36][37][38][39], there is no simple model for the thermal-resistor $\mathrm{SiN}_{\mathrm{x}}$ probes. One reason for this is that the $\mathrm{SiN}_{\mathrm{x}}$ probe is composed of multiple materials with an irregular shape, making any model complex to construct.

In this work, a new membrane-based Johnson noise (JN) calibration device has been designed and fabricated following a novel design strategy targeting the improvement of performance and utility. Two working modes have been demonstrated by both experimental measurement and finite element analysis (FEA), showing an exceptionally uniform temperature distribution across its membrane. By employing the new device, a 1dimensional (1D) heat transfer equation for one commonly used SThM probe type has been developed. This allows the calculation of tip temperature by considering the dominant thermal interactions between the probe and the device: solid-solid contact, water meniscus and air conduction. By matching the calculated tip temperature with real scan results, the thermal interface resistance between the tip and the device is obtained, followed by determination of the thermal contact radius. Finally, a steady-state, lumped-thermal system is described for modelling the probe in air, taking into account heat exchange between the probe, sample and air.

\section{Device Design and Fabrication}

The new JN device is designed to achieve a homogeneous temperature distribution by employing a low thermal conductivity membrane and a central-symmetric configuration of heaters. Figure 1(a) shows an overview of the device. It integrates eight individually addressable heaters, four large and four small, together with a metallic 4terminal thin film resistor, all on a $100 \mathrm{~nm}$ thick $\mathrm{SiN}_{\mathrm{x}}$ membrane. 


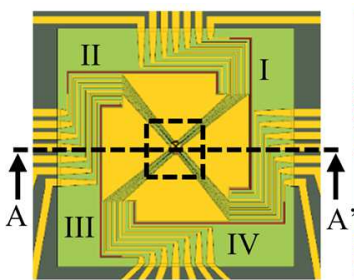

(a)

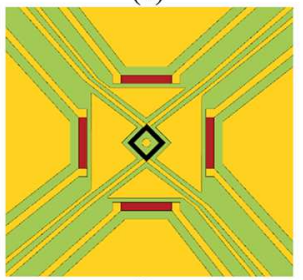

(b)

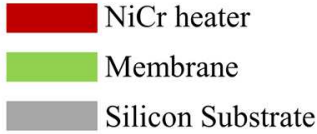

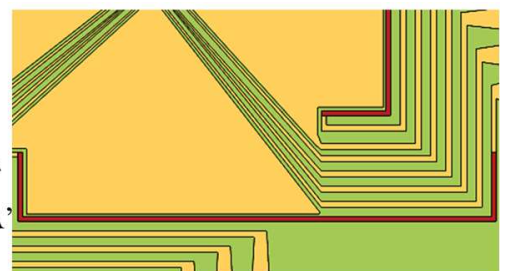

(c)

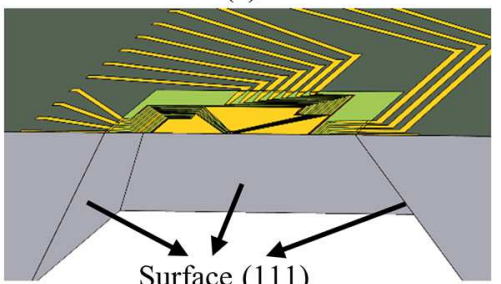

(d)

Membrane on Silicon

Gold

4-terminal resistor

Figure 1. (a) Design of the components on the membrane, (b) close up of one of the large heaters IV, (c) close up of the dashed square in (a), and (d) 3-dimensional cross-section view taken through line A-A' showing the membrane supported by the silicon substrate.

The thin $\mathrm{SiN}_{\mathrm{x}}$ membrane was chosen due to its low thermal conductivity, reducing heat flow away from the device. The same membrane was used by Dobson et al. [15], however, heat loss through asymmetric device wires into the silicon substrate still resulted in some thermal nonuniformity. As a symmetric configuration will lead to maximum temperature uniformity at the centre, multiple heaters were configured with a centralsymmetric layout. It was also necessary to consider heat loss through the gold wires connecting the onmembrane device to the external circuit due to the high thermal conductivity of gold. Thermal uniformity was achieved by employing U-shape heaters, which are marked as I to IV in figure 1(a), to compensate for the heat loss present in the gap between each heater where the device connections exit the membrane. This was designed by optimising the shape of each part using the FEA method, as discussed later. The resulting design feature can be seen as the red U-shape heaters highlighted in figure 1(a) and (c). The inclusion of four small heaters located in the centre of the membrane, shown in figure 1 (b), offered a simple means of altering the size of the heated region and quantifying the effect of air conduction between the cantilever and the sample. Varying the heated region between the whole membrane (named "large heater mode") and the small central part (named "small heater mode") provided a direct measurement of the thermal conduction between sample and tip for a specific temperature distribution. A $2 \mu \mathrm{m}$ diameter, electrically isolated gold circle was fabricated at the centre of the membrane identifying the optimum contact point for the SThM probe. This circle, named the "scanning target", was surrounded by a split 4-terminal resistor (black diamond-shape feature in figure $1(b)$ ). This configuration ensured that the average temperature of the resistor was as close to that of the scanning target as possible. 
The size of membrane was also specially designed for convenience in characterizing the commonly used nanofabricated $\mathrm{SiN}_{\mathrm{x}} \mathrm{SThM}$ probes. The membrane was a $560 \mu \mathrm{m} \times 560 \mu \mathrm{m}$ square and the small heaters bounded a region of $32 \mu \mathrm{m} \times 32 \mu \mathrm{m}$ square. With these dimensions, a heated region covering the size of the whole SThM cantilever (with its length of $150 \mu \mathrm{m}$ ) could be generated using the large heaters, alternatively a region one tenth the probe length could also be created by employing small heaters. Importantly, both of these heating regimes could be generated in a single experiment whilst the SThM probe maintained contact throughout.

The use of Johnson noise to measure temperature requires a thermometer of known resistivity. Therefore, both the heaters and the 4-terminal resistor were made from $\mathrm{Ni}_{0.6} \mathrm{Cr}_{0.4}$, offering a low temperature coefficient of resistance (lower than $0.1 \%$ over the temperature range of the experiments) [40]. The correlation method, as described later, was used to measure the thermal noise of the resistor. The design also included large gold regions located between all of the active features (4-terminal resistor, large and small heaters) as highlighted in Figure 1(a) and (b). These acted as ground planes for electrical isolation and helped spread the heat uniformly across the whole membrane.

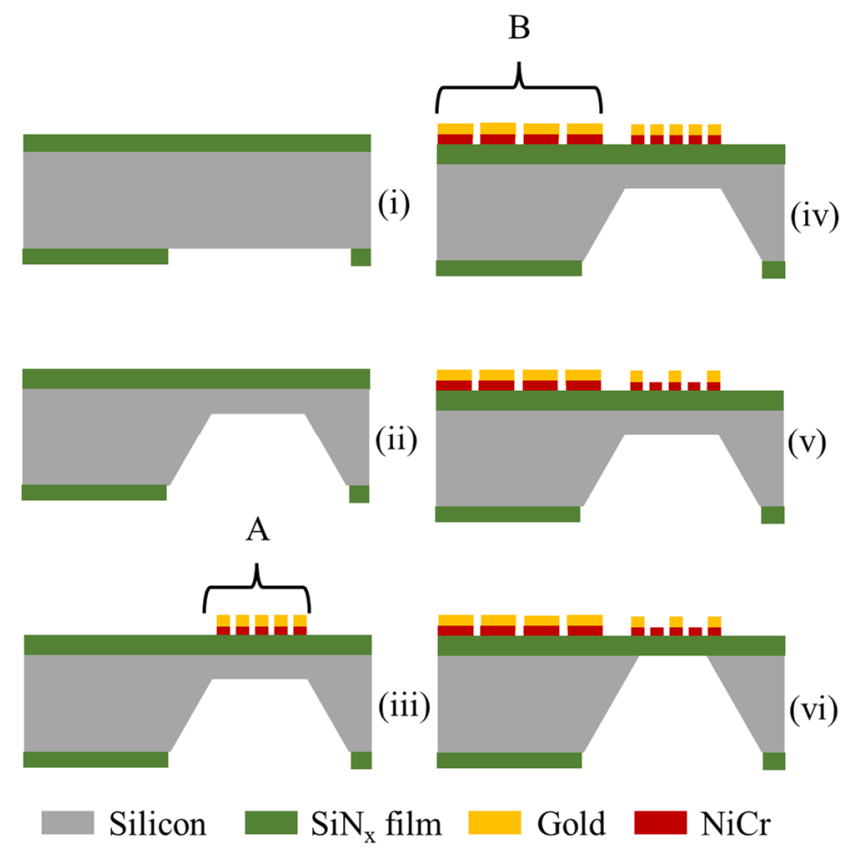

Figure 2. Fabrication process of the JN device in the steps (i) to (vi).

Fabrication of the device was based on a combination of high-resolution electron-beam lithography and photolithography, making the device both highly controllable and economic. The completed fabrication process flow is shown in Figure 2. The JN devices were batch fabricated on a 3-inch $400 \mu \mathrm{m}$ thick n-type (100) silicon wafer with $100 \mathrm{~nm}$ thick LPCVD $\mathrm{SiN}_{\mathrm{x}}$ film on both sides. First, the backside $\operatorname{SiN}_{\mathrm{x}}$ film was removed in lithographically defined regions using reactive ion etching (RIE) with $\mathrm{C}_{2} \mathrm{~F}_{6}$ (step i) [41]. These features were 
used as a wet etch mask to define the membrane. The wet etch employed $7 \mathrm{~mol} \mathrm{KOH}$ at $115{ }^{\circ} \mathrm{C}$ to remove the silicon at a rate of $4.1 \mu \mathrm{m} / \mathrm{min}$. To ensure a robust front face for subsequent fabrication steps, $50 \mu \mathrm{m}$ of silicon was left on the front side (step ii). Next, device features were defined on the topside of the wafer in three steps. For all critical features on the membrane, ebeam lithography using a double layer PMMA resist followed by liftoff was used [42]. The metal was a stack of $165 \mathrm{~nm}$ thick gold with a $15 \mathrm{~nm} \mathrm{NiCr}$ under layer deposited through evaporation in "A" of step iii. For the less dimensionally critical gold wires and bondpads located on the silicon substrate, photolithography was used together with liftoff (S1818-LOR 3A) of the same evaporated metal stack shown in "B" of step iv. Then, a gold etch by KI/I solution was used to expose the underlying NiCr, which would eventually form the heaters and 4-terminal resistor [43] (step v). Finally, the remaining $50 \mu \mathrm{m}$ silicon on the backside was removed in $25 \%$ TMAH mixed with an additional $25 \%$ by volume isopropanol alcohol (IPA) at $80^{\circ} \mathrm{C}$ (step vi). This final silicon wet etch was chosen due to its slow etch rate $(\sim 0.35 \mu \mathrm{m} / \mathrm{min})$ and high selectivity, ensuring no corrosion of other features. The completed devices were cleaved from the silicon wafer for individual use. Scanning electron microscopy (SEM) images of the completed JN device are shown in figure 3.

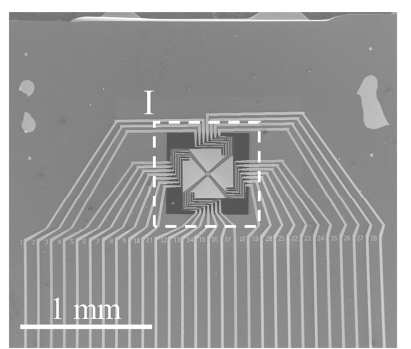

(a)

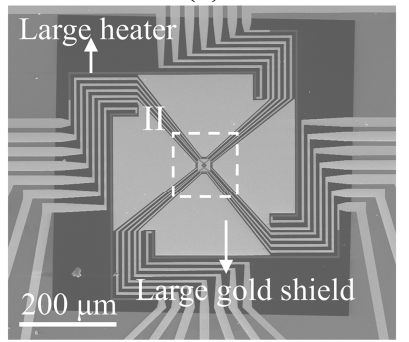

(b)

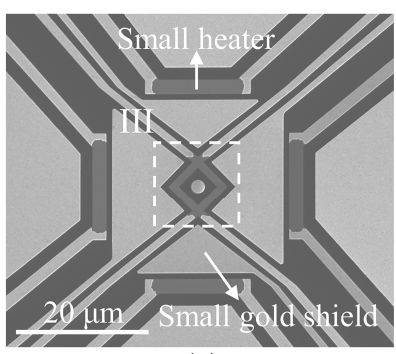

(c)

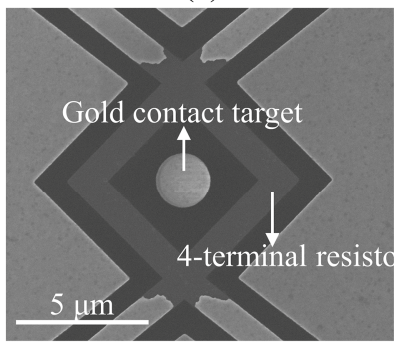

(d)

Figure 3. SEM image of the JN device: (a) general view of the whole device including the membrane (dark grey area) and gold connecting wires (brightest wires), (b) details of features from the dashed square I in figure (a) showing four large heaters and four large gold shields on the membrane, (c) details of features in the dashed square II in figure (b) showing four small heaters and four small gold shields, and (d) details of features in the dashed square III in figure (c) showing the $2 \mu \mathrm{m}$ diameter gold contact target with the $\mathrm{NiCr}$ 4-terminal split resistor surrounding it. 


\section{Experimental Methods}

\subsection{Finite element analysis (FEA) of the JN device}

FEA is commonly used for modelling the temperature distribution of devices at the microscopic scale [22][44]. Considering the complex geometry of the device, a 3D model was used in order to simultaneously account for its multiple materials, heat exchange with surrounding environment, and steady-state operation. As most elements in the device are thin films, thermal transport will be affected by their smallest dimension, which is comparable in length to the MFP of heat carriers. Therefore, thermal conductivities of materials in the device needed to be corrected to take this size effect into account.

For metals where values where not readily available in the literature, it was necessary to estimate their thermal conductivity. This was done from their electrical conductivity using the Wiedemann-Franz (W-F) Law [45], which states that the ratio of thermal conductivity and electrical conductivity is a function of temperature:

$$
\frac{k}{\sigma}=c T
$$

where $k$ is thermal conductivity, $\sigma$ is electrical conductivity, $c=2.45 \times 10^{-8}$ is a constant named the Lorenz number, and $T$ is the temperature in kelvins. As the resistance of the $\mathrm{NiCr}$ small heater can be measured as 792 $\Omega$, and its dimensions are known, the electrical conductivity can be calculated as $5.41 \times 10^{5} \mathrm{~S} / \mathrm{m}$. Therefore, WF gives a thermal conductivity for our thin-film $\mathrm{NiCr}$ at $300 \mathrm{~K}$ as $5.1 \mathrm{~W} / \mathrm{mK}$. The thermal conductivity of the gold used in wires and shields, as well as that of the LPCVD $\operatorname{SiN}_{\mathrm{x}}$ membrane was taken from the literature for films of the same thickness and composition [46][47]. The silicon wafer (400 $\mu \mathrm{m}$ thick) was considered as bulk silicon. Table 1 shows the thermal conductivity used for each material in the 3D model.

Table 1. Thermal conductivity of each element in the JN device

\begin{tabular}{llll}
\hline Element & Material & $\begin{array}{c}k \\
(\mathrm{~W} / \mathrm{mK})\end{array}$ & Source \\
\hline $\begin{array}{l}\text { Substrate } \\
\text { Membrane }\end{array}$ & Silicon & 130 & $\begin{array}{l}\text { Bulk, } \\
\text { intrinsic }\end{array}$ \\
$\begin{array}{l}\text { Wires, shields, } \\
\text { and scanning } \\
\text { target }\end{array}$ & Gold & 317 & Ref. [47] [46] \\
$\begin{array}{l}\text { Heaters and } \\
\text { thermistor }\end{array}$ & $\mathrm{Ni}_{0.4} \mathrm{Cr}_{0.6}$ & 5.1 & W-F Law \\
\hline
\end{tabular}


A steady state model taking both Joule heating and heat transfer into account was built in COMSOL MULTIPHYSICS ${ }^{\circledR}$. As the device was used under ambient conditions, the model included a surrounding block of material with the thermal properties of air. All elements of the device were modelled with the same dimensions as those fabricated, however in the interest of computational efficiency the model only extended $600 \mu \mathrm{m}$ beyond the membrane, at which point an ambient boundary condition was set since the silicon substrate has much larger thermal conductivity than the silicon nitride membrane and was thermally grounded to the microscope. All inner boundaries were set to have continuity of temperature, and all outer surfaces, including those of the device and the air, were fixed at room temperature ( $293 \mathrm{~K})$. The initial conditions for the model were also set to room temperature. As heaters were employed in parallel during the experimental work, electrical boundary conditions were set the same for each, with an electrical potential on one lead and ground at the other.

\subsection{SThM setup for temperature measurement}

The device was scanned using nanofabricated, thermally compensated probes developed in Glasgow by Zhang et al. [35]. The probe was used in passive mode as a nano-thermometer allowing measurement of the temperature distribution on the membrane. As the JN device was heated using a DC voltage, care was taken to avoid an unintended direct electrical path between the device and probe instrumentation. This was achieved by employing the method described by Dobson et al.[48], the schematic of which is shown in Figure 4. The probe was connected in one leg of a Wheatstone bridge and driven by a $2.5 \mathrm{MHz} 140 \mathrm{mV}$ peak-peak sinusoid wave through a transformer. Bodzenta et al. [49] observed that thermal sensitivity of a SThM probe varied significantly when the frequency of its driving current exceeded a cut-off value $(\sim 10 \mathrm{kHz})$. In contrast, the method employed in this work employed very small RF transformers mounted in close proximity to the probe, minimising the stray capacitance connecting the probe and external circuitry. The measurement itself was of changes in probe resistance, which are substantially at DC. It should also be noted that no significant thermal effects were observed due to heating of the probe at radio frequencies. In this configuration, the current passing through the probe was no larger than $230 \mu \mathrm{A}$. Previous work by Dobson [48] has shown that significant probe self-heating will not occur until the AC current exceeds $1 \mathrm{~mA}$. Therefore, self-heating of the probe could be regarded as negligible during the experiment. When used as a thermometer, the resistance of the platinum tip changes with temperature, leading to a change in the output of the bridge. This was connected to a lock-in amplifier through another transformer and then compared to a reference signal. The use of phase synchronous 
detection allowed the significant contribution of SThM probe pad capacitance to be eliminated by the choice of a suitable measurement phase. This worked because the thermal signal affects the DC resistance of the probe only, and is therefore in quadrature to the parasitic signal from the reactive impedance of the pads. The DC output voltage from the lock-in amplifier was then fed into the AFM (Veeco Dimension 3000) control unit via a signal access module. This configuration permitted measurement of electrically live samples without requiring dielectric coatings, which increase sample complexity and decrease the accuracy of the measured temperature. A more detailed description of this setup can be found in [48]. In order to quantify the output signal, the probe's electrical resistance $\left(R_{p}\right)$ was obtained $i n$-situ from the adjustable balanced resistor $R_{M}$, after which the variation of $R_{p}$ instead of its absolute value, was used.

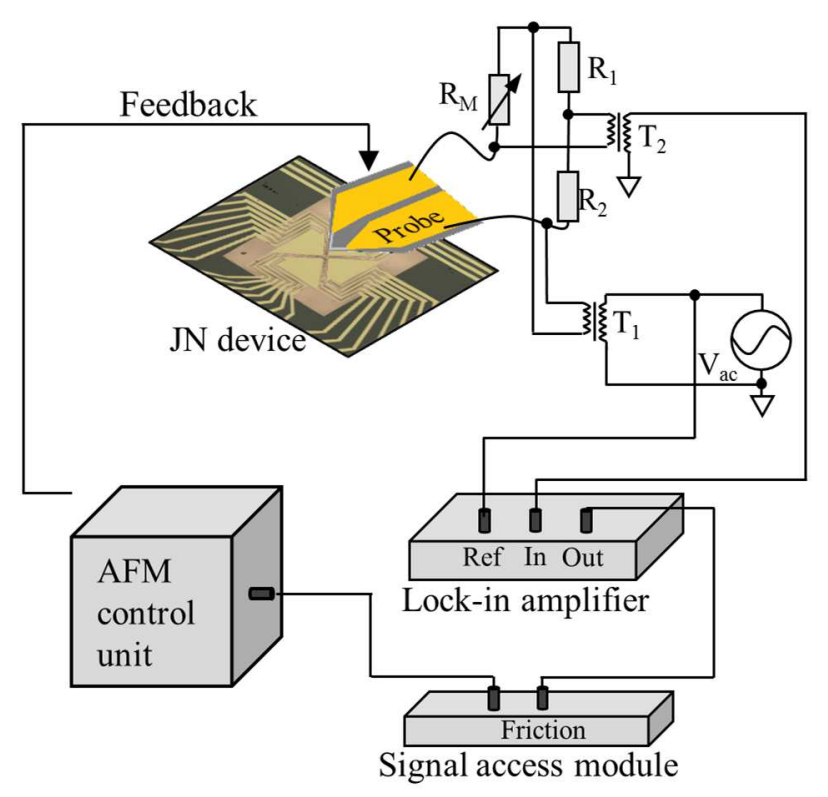

Figure 4. Schematic of the experimental setup for a passive SThM probe scanning of the in-situ JN device. The probe forms a Wheatstone bridge with $R_{M}, R_{1}$, and $R_{2} . T_{1}$ and $T_{2}$ are transformers used for exciting and measuring the output of the bridge.

The temperature variation of the probe can be determined from the output voltage $(U)$, from which probe resistance is derived using parameters of the circuit (peak-peak AC voltage supplying the bridge, $V_{a c}$ ) and gain of the lock-in amplifier $(A)$. We have previously shown that the temperature coefficient of resistance $(\alpha)$ of the whole probe, consisting the cantilever, gold wires, pads, and the platinum tip, is $0.000961 \pm 0.0000106 \mathrm{~K}^{-1}$ [50]. Thus, the real temperature variation on the probe can be calculated as:

$$
\Delta T_{p}=\frac{2 m}{(1-m) \alpha}
$$

where $m=2 U /\left(V_{a c} A\right)$. 


\section{Results and Discussion}

\subsection{Experimental verification of the FEA model and probe calibration}

The JN device was tested in two modes, with only the large heaters or only the small heaters active. The unbiased 4-terminal resistor at the centre of the device was connected to two identical differential amplifiers based on the OPA637 uncompensated DIFET input operational amplifier in a standard instrument amplifier configuration. A correlation measurement of the output of the two amplifiers allows a measure of the thermal noise of the 4-terminal resistor, which is independent of the (uncorrelated) voltage noise contributions of the two amplifiers. The correlation circuit allowing for measurement of Johnson noise and its conversion into absolute temperature is a simple analogue correlator as used in [15]. Electrical resistance of the small and large heaters was $792 \pm 1.1 \Omega$ and $11005 \pm 1.3 \Omega$ respectively, as determined from 10 devices. This variation in resistance will result in a less than $1 \%$ difference in the power from each heater, with negligible effect on the temperature distribution of the membrane. Previous work has shown that the random variation uncertainty in the system is $\pm 0.73 \mathrm{~K}$ [15]. The FEA model was verified by simulating a JN device with defined heater power (figure 5 (a) and (b)), and then the same power was used to heat a real device allowing the simulated and experimental 4-terminal resistor temperatures to be compared (figure 5 (c) and (d)). The heaters were biased using a simple DC voltage source based on a voltage reference and 10-turn potentiometer, with extensive filtering to prevent capacitive coupling of noise into the system within the bandwidth of the correlator. Validation of the model is demonstrated by the agreement between the simulation and experiment. Analysis of the validated FEA result shows that at $80 \mathrm{~K}$ above ambient for small heater mode and at $150 \mathrm{~K}$ above ambient for large heater mode, the gold scanning target has less than $0.5 \mathrm{mK}$ difference with the average temperature of the 4-terminal resistor. The uniformity of the temperature at the central region of the membrane is shown to be better for the large heaters than the small heaters, a phenomenon attributed to the different distance between each and the centre. 


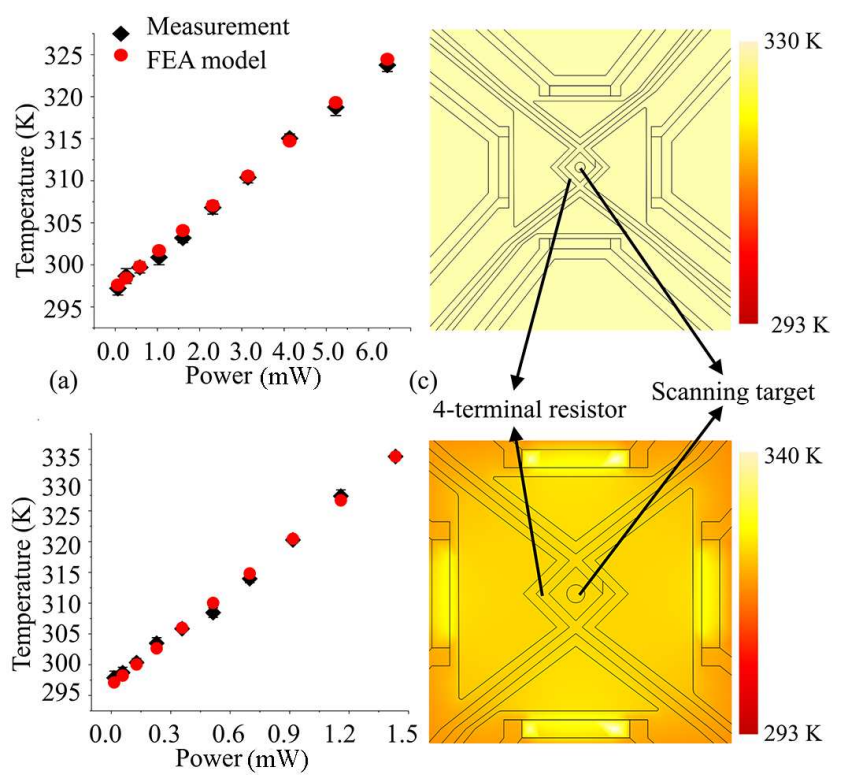

(b)

(d)

Figure 5. Plot of the average temperature on the 4-terminal resistor versus the power on heaters obtained from the experiment (black diamonds) and FEA model (red circles) for the JN device working in (a) large heaters and (b) small heaters mode; (c) and (d) show the temperature distribution at the centre of the membrane under high heating.

The primary purpose of the $\mathrm{JN}$ device is to offer a sample with well-known temperature and thermal distribution for use in calibrating SThM probes. The instrumentation used to achieve this was set up as described in 3.2. The SThM probe was brought into contact with the JN device and positioned on the scanning target. The temperature of the $\mathrm{JN}$ device $(T)$ and the output from the probe $(U)$ were then recorded for various heater powers. Figure 6 shows the probe calibration plots obtained using both the small and the large heaters. A least squares method was used to fit the data to a straight line ( $T$ - $U$ line), relating the probe signal to the sample temperature. For small heater mode, $T_{1}=(26.365 \pm 0.2201) U_{1}+292.79 \mathrm{~K}$ and for large heater mode, $T_{2}=(17.083 \pm$ $0.1025) U_{2}+293.26 \mathrm{~K}$. 


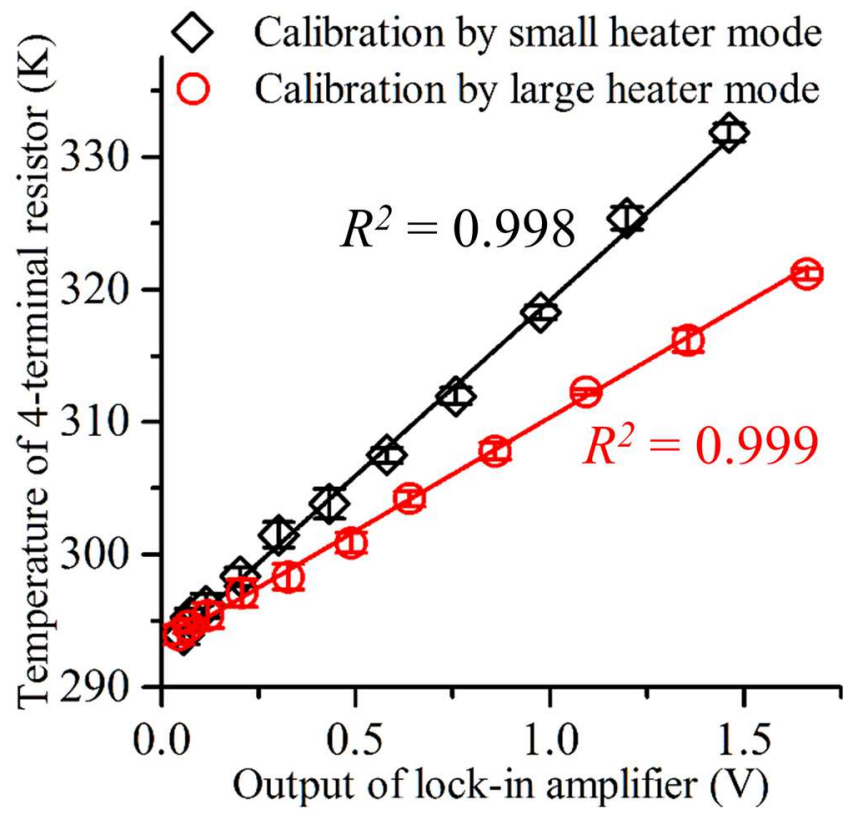

Figure 6. The temperature of the 4-terminal resistor on the JN device versus the DC output of the lock-in amplifier for the small heater (black squares) and large heater (red circles). Parameters of the linear fit are shown with the $R^{2}$ value. The error bars are one standard deviation as obtained from the random variation in the data points.

It can be clearly seen in Figure 6 that a change in the size of the heated region, whilst maintaining the same temperature at the contact point, can result in a large variation in the output from the probe when calibrating in air. This difference can be significant, in this instance being more than 40 times larger than the error in the JN device. This difference can be attributed to the effect of through-air conduction between the cantilever and the sample as observed by other groups [12][25][51]. One solution is to employ a high vacuum system. However, the complicated setup and high cost associated with a vacuum system preclude its use in many situations. Therefore, it is essential that a clear understanding of probe-sample interactions be employed during the analysis of experimental results in order to avoid misinterpretations.

\subsection{D heat transfer model for probe-JN device interactions in air}

It is well recognized that there are multiple thermal paths between a SThM probe and sample, and that solidsolid direct contact, water meniscus and air conduction dominate, while thermal radiation is typically negligible [1][12][52]. For a probe working in passive mode, net heat flow is governed by thermal conduction within the probe, through the surface contact point (by the solid-solid contact and water meniscus), and through air between the probe and the sample. These combined heat paths can be analyzed by taking into account a heat transfer model constructed and extended by Shi et al. [25] and Kim et al. [53] respectively, as an equation:

$$
\frac{d}{d x}\left[\frac{d T(x)}{d x} \sum_{i}[k A(x)]_{i}\right]+h(x) w(x)\left[T(x)-T_{s}(x)\right]=0
$$


where $k$ is the thermal conductivity, $A$ is the cross section area, $w$ is the width of the cantilever that participates in heat exchange with the sample, $\mathrm{h}$ is the heat transfer coefficient varying with probe-sample separation, $T$ and $T_{s}$ are the temperature of the probe and sample. Solving probe temperature from (3) requires parameters of the probe-sample interaction system. With the probe's well-controlled geometry, the same as those used in previous work by Assy et al. [3],[54] and Puyoo et al.[34],[55], this model can be transferred for use when scanning the JN device. Due to its complex geometry and composition of multiple materials, the probe was split into subregions as denoted by subscript $i$ in (3).

As shown in figure 7 , the probe was mounted with the cantilever at a $13^{\circ}$ angle to the sample surface $(\beta)$, while the tip was at a $69^{\circ}$ angle $(\varphi)$ to the sample. At the contact point $(x=0)$, heat transfers from the sample surface to the probe through the tip-surface contact with a thermal conductance defined as $G_{t s}$. At the base of the cantilever, $x=L$, the temperature was assumed to be ambient $T_{R T}$, as the silicon base is treated as a perfect heat sink in common with other studies $[3,22,55]$. Therefore, two boundary conditions at $x=0$ and $x=L$ are:

$$
\begin{aligned}
G_{t s}\left[T_{s}(0)-T(0)\right] & =\sum_{i}[k A(L)]_{i} \frac{d T(L)}{d x} \\
T(L) & =T_{R T}
\end{aligned}
$$

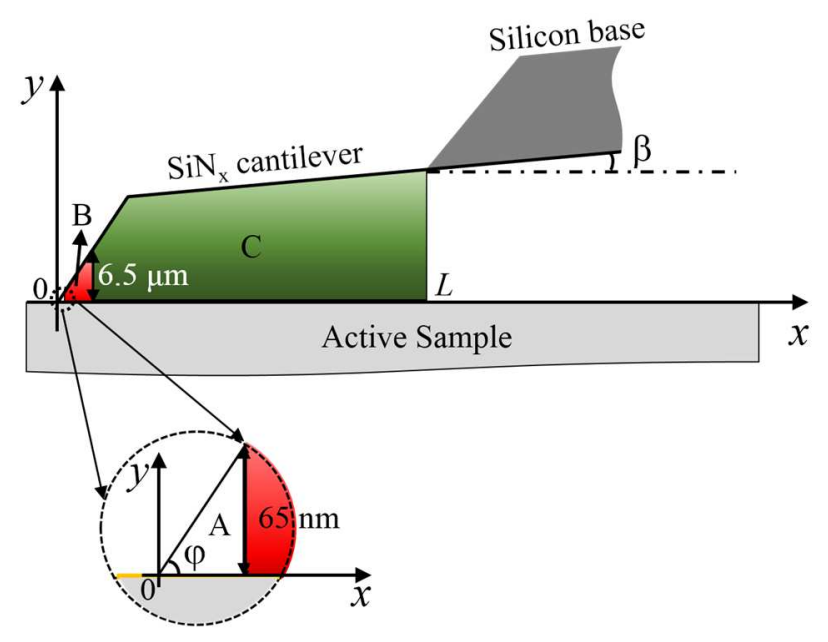

Figure 7. Schematic diagram shows the geometry of the passive probe scanning an active sample. Three regions of heat exchange between the probe and sample through air conduction are determined by the separation and are shown as A, B and C.

Solving the equation requires knowledge of various thermal properties within the system. The gold on the probe was the same thickness as that on the $\mathrm{JN}$ device, therefore the thermal conductivity was taken to be the same as that in table $1(317 \mathrm{~W} / \mathrm{mK})$. For the $500 \mathrm{~nm}$ thick $\mathrm{SiN}_{\mathrm{x}}$ cantilever, thermal conductivity was taken from the literature as $10 \mathrm{~W} / \mathrm{mK}$ [56] and for the platinum tip resistor, the thermal conductivity was calculated using $\mathrm{W}-\mathrm{F}$ as $22 \pm 1.1 \mathrm{~W} / \mathrm{mK}$. Conduction through the air is dictated by the ratio of the distance $(\delta)$ to the MFP of air 
$(\lambda=65 \mathrm{~nm})$, and was therefore divided into three regimes, ballistic, slip and bulk respectively [25]. It was also assumed that air conduction was independent of materials at the surface of probe or JN device [57],[58]. Corrected thermal conductivities were then converted into heat transfer coefficients $(h)$ by using the appropriate distance, $\delta(x)$ as dictated by the geometry.

By substituting derivative terms for finite differences, (3), (4) and (5) were transferred from ordinary differential equations (ODEs) into a set of simultaneous algebraic equations [59], which were then implemented in MATLAB. This enabled the temperature distribution as calculated using COMSOL to be related to the signal output of the SThM probe. In order to fully account for the probe-sample heat exchange through the air, it was necessary to provide the equation with the discrete actual temperature distribution (as modelled in COMSOL) for the device in the region below the probe. The only unknown parameter was the tip-sample thermal conductance, $G_{t s}$, which was determined by varying the value until an appropriate fit between experimental results and MATLAB modelled data was obtained.

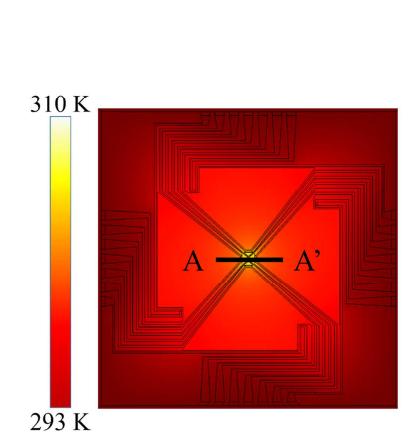

(a)

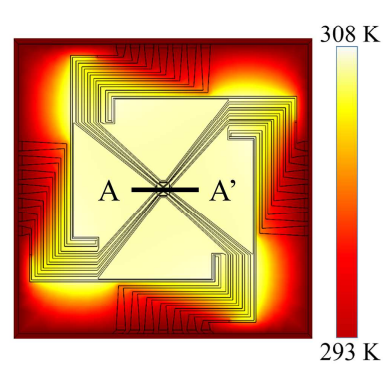

(b)

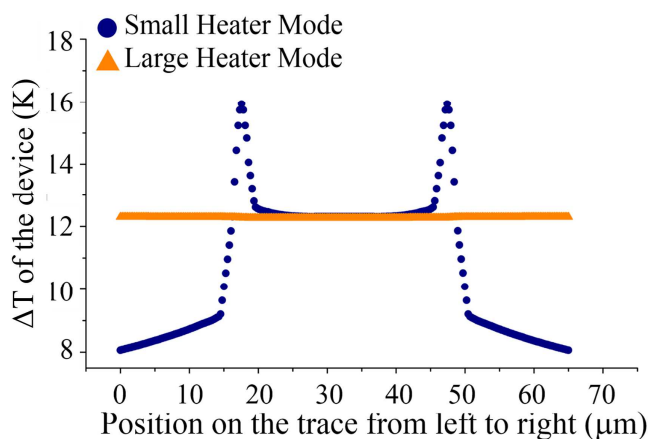

(c)

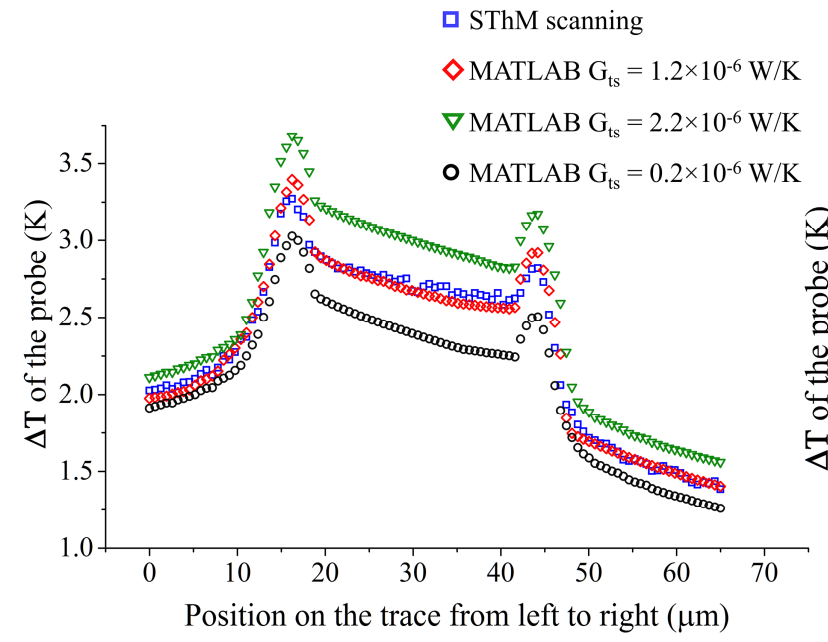

(d)

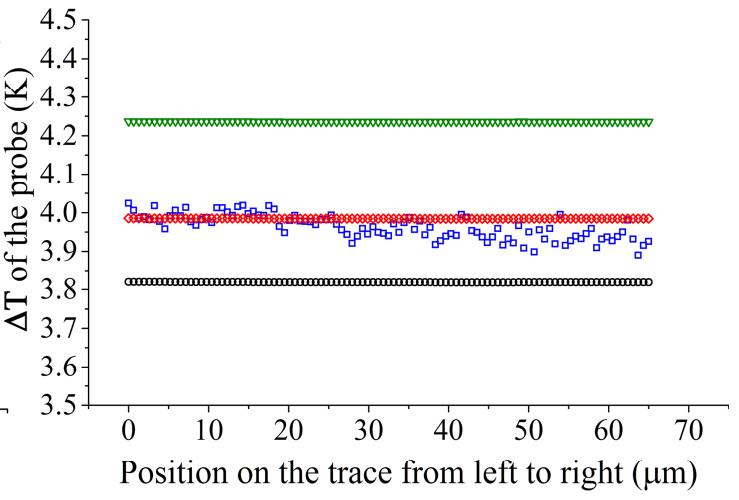

(e)

Figure 8. (a) and (b) show JN device temperature distribution from the FEA model. A $65 \mu \mathrm{m}$ long trace A-A' across the centre of the membrane shows the section used for the data plotted in (c) showing temperature difference from ambient. (d) and (e) show a comparison, for the same region, between SThM acquired experimental data (blue squares) and modelled data corrected using the heat transfer equation with different $\mathrm{G}_{\mathrm{ts}}$ values (red diamonds, green triangles and black circles), corresponding to (a) and (b). 
Figure 8 shows the FEA results of the temperature distribution on the membrane when heated by small (a) and large (b) heaters. Heating powers were selected to obtain the same temperature on the 4-terminal resistor in each case: $360 \mu \mathrm{W}$ - small heaters and $2000 \mu \mathrm{W}$ - large heaters. For each simulation, the temperature distribution profile was extracted along a $65 \mu \mathrm{m}$ line A-A' as shown in (c). This was the same as the scan length and location used to obtain the experimental data. It can be seen that, in accordance with design intentions, the temperature distribution at the membrane is uniform for the large heaters, whereas, the small heaters produce a much smaller isothermal region. The actual temperature of the probe acquired by experiment was much smaller than the sample temperature in both modes (blue squares figure 8 (d) and (e) compared with (c)). It also exhibited an asymmetric distribution most clearly observed for the small heaters. This artefact is considered to be the result of varying heat flow through the air from a non-uniform sample temperature. This is confirmed by the excellent fit obtained by correcting the FEA data (figure 8 (c)) using the MATLAB code as shown by the red diamonds in figure $8(\mathrm{~d})$ and (e). This fit was achieved with a tip-sample thermal conductance $G_{t s}=1.2 \times 10^{-6} \mathrm{~W} / \mathrm{K}\left(R_{t s}=\right.$ $8.33 \times 10^{5} \mathrm{~K} / \mathrm{W}$ ). The main error in the model is due to the assumption of room temperature at the end of the cantilever connected to the silicon base. This can be seen in figure 8 (e) where the experimental results show a slight gradient that we attribute to variation in the temperature of the silicon probe base as the probe is slowly scanned over the large heated region.

\subsection{Analysis of $G_{t s}$}

Unlike thermal conduction along the probe and through the air, $G_{t s}$ is a value attributed to multiple heat paths at the probe-sample constriction. The constriction thermal resistance $\left(R_{\text {constriction }}\right)$ is a combination of thermal resistance due to the solid-solid contact $\left(R_{\text {int }}\right)$, the constriction resistance for the tip side $\left(R_{\text {tip }}\right)$ and sample side $\left(R_{s}\right)$, the water meniscus $\left(R_{w}\right)$ and the air surrounding the constriction. It has been experimentally demonstrated by Assy et al. [52][54] that for the probes used in this work thermal conductance of the water meniscus is $1 \%$ $-6 \%$ of total contact conductance, suggesting that this effect can be neglected. Size of the mechanical contact between the probe and the sample has previously been determined using the DMT model [33]. For a contact force of $20 \mathrm{nN}$, as used in this work, the contact radius is $3 \pm 0.5 \mathrm{~nm}$ [3]. With this information, it is possible to model the tip as a hemisphere connected to the triangular probe end as shown in figure 9 (a) with an associated resistance model shown in figure 9 (b). When considering a heated sample, the probe contact can be regarded as an isothermal system, as demonstrated by the work of Fletcher et al. [30] using a thermoelectric probe. 
Therefore, we can assume that the temperature of surface $1\left(T_{\text {surface }}\right)$, surface $2\left(T_{\text {surface } 2}\right)$ and the interface ( $T_{\text {interface }}$ ) are all equal. Therefore, $R_{s}$ does not need to be considered, as no thermal spreading exists. The constriction thermal resistance $\left(R_{\text {constriction }}\right)$ can be then calculated as:

$$
R_{\text {constriction }}=R_{\text {int }}+\sum_{j}\left(R_{\text {air }}^{j} \| R_{\text {hemi }}^{j}\right)
$$

where $j$ represents the number of elements in parallel, which is infinite.
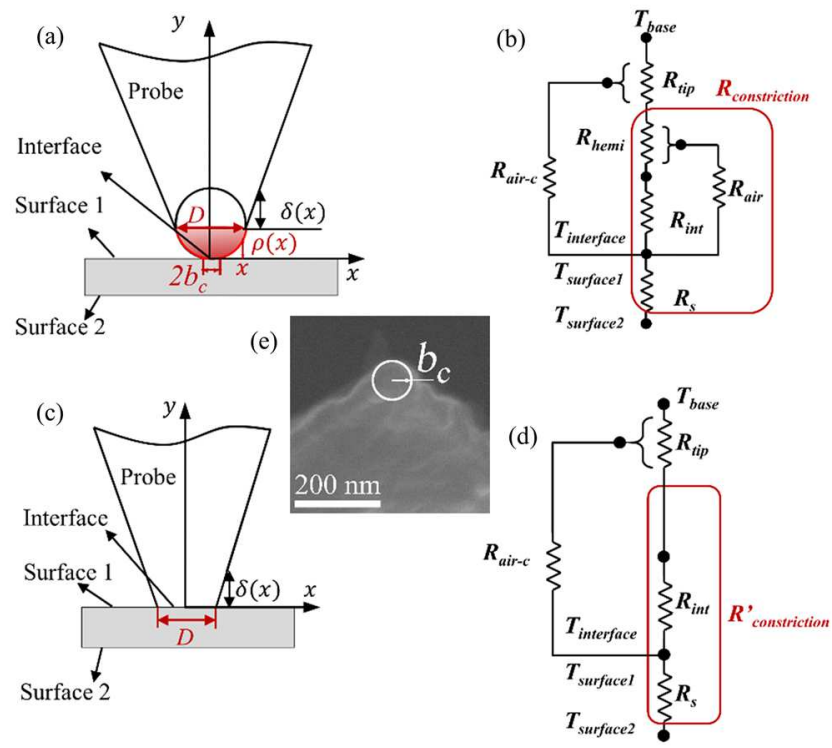

Figure 9. (a) Constriction model between the tip and surface based on the DMT model determined contact radius, (b) associated thermal resistance network, (c) constriction model between the tip and surface with the flattened apex equal to the contact radius and (d) associated thermal resistance network. (e) $10 \mathrm{kV}$ SEM image of probe apex captured in low vacuum mode using a FEI Nova 630.

$R_{h e m i}$ can be determined as $1.04 \times 10^{6} \mathrm{~K} / \mathrm{W}$ assuming that the temperature is a function of the radius by:

$$
R_{\text {hemi }}=\frac{\int_{y_{1}}^{y_{2}} \frac{1}{\pi\left(D y-y^{2}+b_{c}^{2}\right)} d y}{k_{p t}}
$$

where $k_{p t}$ is the thermal conductivity of the platinum, $22 \mathrm{~W} / \mathrm{mK}$ as calculated previously, and $y_{1}$ and $y_{2}$ are 0 and $50 \mathrm{~nm}$ respectively. As the maximum distance between any point on the hemisphere and the sample surface is smaller than the MFP of air at room temperature, thermal transport through the gap is ballistic and heat flow can be written as:

$$
Q_{\text {air }}=2 \pi k_{\text {air }-b} \int_{b_{c}}^{\frac{D}{2}} x \frac{\Delta T(x)}{\rho(x)} d x
$$

This gives the $R_{\text {air }}$ at the tip as $1.09 \times 10^{9} \mathrm{~K} / \mathrm{W}$.

Finally, $R_{\text {int }}$ is determined using the DMT model as:

$$
R_{\text {int }}=\frac{R_{b}}{\pi b_{c}^{2}}
$$


where $R_{b}$ is the thermal boundary resistance $\left(10^{-9}\right.$ to $5 \times 10^{-8} \mathrm{~m}^{2} \mathrm{~K} / \mathrm{W}$ [26][60]), giving $R_{\text {int }}$ in the range $2 \times 10^{7}$ to $10^{9} \mathrm{~K} / \mathrm{W}$.

Using these calculated values in (6), $R_{\text {constriction }}$ is determined to be larger than $2 \times 10^{7} \mathrm{~K} / \mathrm{W}$. This is at least two orders of magnitude larger than the $R_{t s}$ required to obtain a good fit to the experimental data as shown in Figure 8 (d) and (e). Furthermore, recent work by Puyoo [34], Gotsmann [61], Pettes [23] and Assy [3], suggests it may be more appropriate to consider the contact radius to be the same as the probe's tip radius of curvature. In this instance, the thermal resistance model can be modified as shown in figure 9 (c) and (d). The tip is regarded as a cone with a round, planar contact to the surface of radius $\sim 50 \mathrm{~nm}$, which makes the thermal constriction resistance $R_{\text {constriction }}$ simply given by $R_{\text {int }}$. Therefore, $R_{b}$ can be calculated as $6.5 \times 10^{-9} \mathrm{~m}^{2} \mathrm{~K} / \mathrm{W}$ by using $R_{\text {int }}=R_{t s}$ $=8.33 \times 10^{5} \mathrm{~K} / \mathrm{W}$ and $b_{c}=50 \mathrm{~nm}$. This value is in good agreement with the thermal resistance of a metal-metal interface $\left(10^{-9} \mathrm{~m}^{2} \mathrm{~K} / \mathrm{W}\right)[30][62]$. It should be noted that $\mathrm{SThM}$ probes always have a rough tip apex and tend to establish a multi-asperity contact. In this model, this complicated contact scheme was simplified to a single contact for two reasons: on the larger scale, there were no obvious multi-contact artefacts visible in the topographic image; on the smaller scale, it has been shown by Gotsmann's work that even a rough tip should be treated as flattened due to the pressure exerted by imaging and that heat transport will increase as a function of true contact area [61]. This is an effective approach as demonstrated in other research employing SThM probes [3][23][54].

\subsection{Application of the JN device for characterizing the SThM probe}

Unlike the single-material, doped silicon or Wollaston probes for which thermal resistance can be directly calculated from their dimensions and thermal conductivity [36][37][38], the SThM probe used in this work is difficult to characterize due to its complex, multiple-material structure. Current methods for modelling this probe are either too difficult to apply because of their heavy calculation load [63] or inaccurate [64]. There is also considerable effort required when measuring the thermal resistance of the probe as this can only be carried out in vacuum [3]. With the uniform thermal distribution and accurately known temperature, the thermal behaviour of the probe can be determined using the JN device in air. This permits the construction of a lumped system model that describes the thermal behaviour of the probe. This model may provide a simple way to evaluate the characterization of the probe and assist in modifying its design. 
The multiple materials, complex geometry and various probe-sample thermal conduction regimes require a model consisting of five parts, which are shown in figure 10 (a) to (e). One advantage of our probe is that when mounted in an AFM, the ballistic and slip regimes only have impact on the platinum sensor, reducing the number of parts to model. Parts $\mathrm{A}$ and $\mathrm{B}$ consist of $\mathrm{SiN}_{\mathrm{x}}$ with platinum in the ballistic and slip regimes respectively. Part $\mathrm{C}$ is $\mathrm{SiN}_{\mathrm{x}}$ with gold on the $69^{\circ}$ angled tip region. Part D and $\mathrm{E}$ are $\mathrm{SiN}_{\mathrm{x}}$ and gold, each with differing geometries on the $13^{\circ}$ inclined cantilever. Each part requires its individual thermal resistance to be determined. Fin theory, as previously used for this probe type, is invalid here due to its requirements for an invariable shape and known thermal conductivity. However, temperature uniformity can still be assumed, as the Biot number is much smaller than 1 [65]. The thermal resistance of the cantilever, accounting for its variable cross section (figure 10 (f)), can be written as:

$$
R_{i}=\frac{\Delta T}{Q}=\frac{\int_{x_{1}}^{x_{2}} \frac{d x}{A(x)}}{k_{i}}
$$

where thermal conductivity $k_{S i N}$ is for $\mathrm{SiN}_{\mathrm{x}}, k_{A u}$ is for gold and $k_{p t}$ is for the platinum thermometer, all as determined previously.
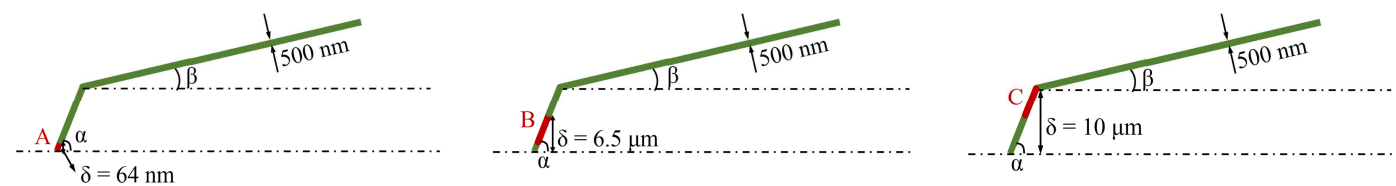

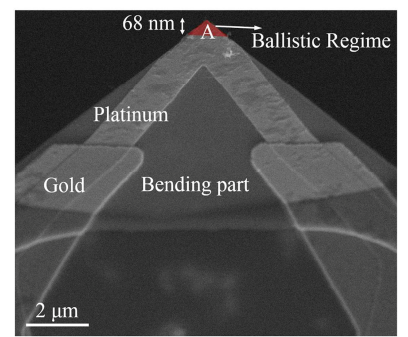

(a)
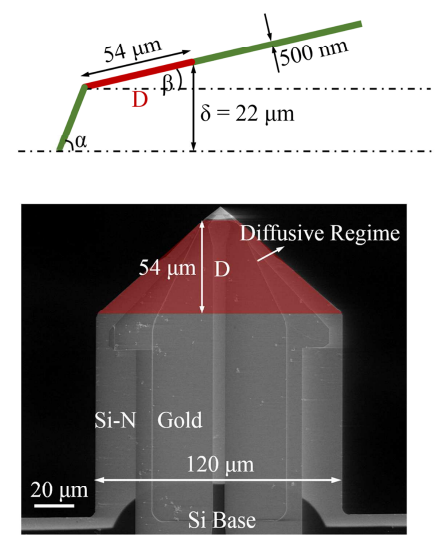

(d)

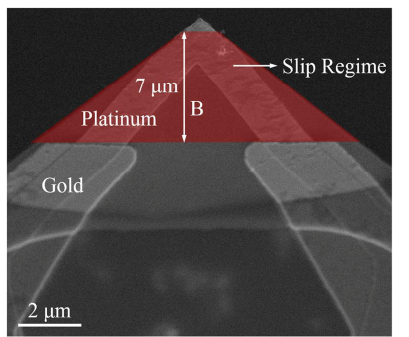

(b)
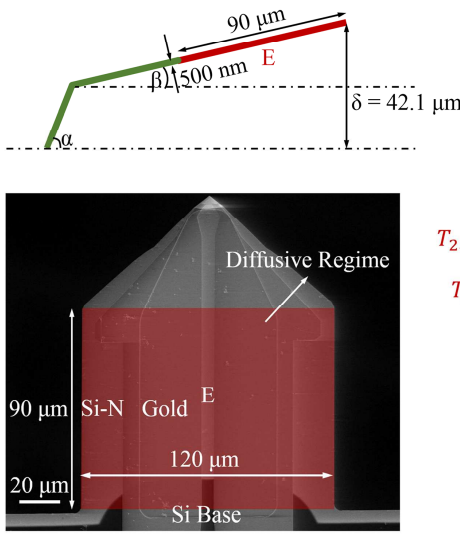

(e)

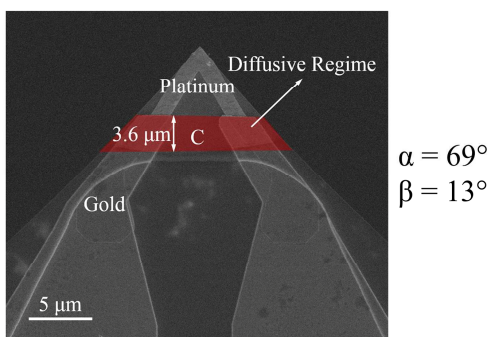

(c)

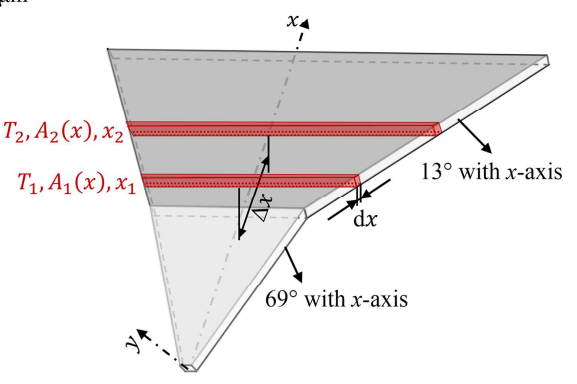

(f)

Figure 10. (a) - (e) show the 5 parts A -E of the probe, each having different dimensions, material and air conduction regimes, and (f) model used for calculating the heat flow thermal resistance through the probe. 
Equation (8) can be used to calculate the thermal resistance of the air between the probe and sample by substituting the perimeter of the hemisphere with the width of the cantilever. Finally, the thermal convective resistance $R_{\text {conv }}$ between the probe and the air is given by:

$$
R_{\text {conv }}=\frac{1}{h_{\text {air }}}
$$

where $h_{\text {air }}$ is the heat transfer coefficient for natural convection which is generally lower than $10 \mathrm{~W} / \mathrm{m}^{2} \mathrm{~K}$ [65], and $S$ is the surface area of each part.

To generate the lumped model, each part of the probe is represented by three components: probe-sample thermal resistance due to air conduction $\left(R_{i}^{\text {air }}\right)$, resistance due to heat flow along the cantilever $\left(R_{i}^{\text {material }}\right)$ and resistance due to air convection $\left(R_{i}^{c o n v}\right)$. Heat capacities are neglected from the network as the model only considers steady-state interactions. Figure 11 shows the resistance network of the probe contacting the gold scanning target when the JN noise device is heated by large heaters, ensuring that the temperature beneath the whole probe is uniform and accurately known. Temperature of the JN device is represented by the electric potential: with ground being room temperature $T_{R T}$, and positive voltages $U_{t h}$ being a temperature above the ambient. Five individual probe parts are shown with part A and B in detail, while part C to E are the same as A and B, but replacing $R_{i}^{p t}$ with $R_{i}^{A u}$. Values of all components used, and associated equations involved, are shown in table 2.

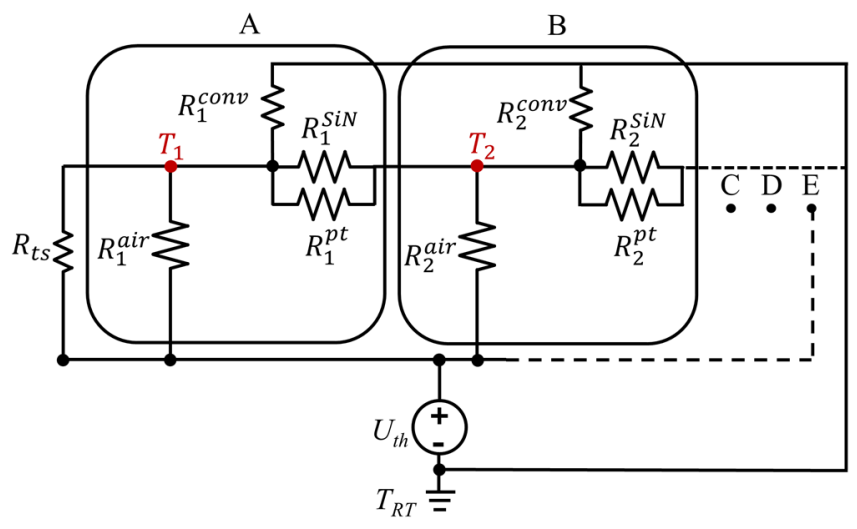

Figure 11. Thermal resistance network for the lumped model of the probe contacting a heated surface. The voltage source represents the temperature of the $\mathrm{JN}$ device, in this instance providing a uniform temperature beneath the probe cantilever. 
Table 2. Values of each thermal resistor in the lumped-system.

\begin{tabular}{llllll}
\hline $\begin{array}{l}\text { Thermal resistance } \\
\text { for }(\mathrm{K} / \mathrm{W})\end{array}$ & $\mathrm{A}$ & $\mathrm{B}$ & $\mathrm{C}$ & $\mathrm{D}$ & $\mathrm{E}$ \\
\hline Air conduction $^{\mathrm{a}}$ & $R_{1}^{\text {air }}=6.383 \times 10^{9}$ & $R_{2}^{\text {air }}=6.99 \times 10^{6}$ & $R_{3}^{\text {air }}=1.192 \times 10^{7}$ & $R_{4}^{\text {air }}=1.404 \times 10^{6}$ & $R_{5}^{\text {air }}=9.819 \times 10^{4}$ \\
$\mathrm{SiN}_{\mathrm{x}}{ }^{\mathrm{b}}$ & $R_{1}^{S i N}=3.046 \times 10^{4}$ & $R_{2}^{S i N}=1.468 \times 10^{5}$ & $R_{3}^{S i N}=1.492 \times 10^{4}$ & $R_{4}^{S i N}=1.732 \times 10^{5}$ & $R_{5}^{S i N}=1.462 \times 10^{5}$ \\
$\mathrm{Pt}^{\mathrm{b}}$ & $R_{1}^{p t}=3.14 \times 10^{5}$ & $R_{2}^{p t}=3.15 \times 10^{6}$ & - & - & - \\
$\mathrm{Au}^{\mathrm{b}}$ & - & - & $R_{3}^{A u}=3.22 \times 10^{4}$ & $R_{4}^{A u}=3.31 \times 10^{4}$ & $R_{5}^{A u}=2.37 \times 10^{4}$ \\
Air convection $^{\mathrm{c}}$ & $R_{1}^{\text {conv }}=8.94 \times 10^{12}$ & $R_{2}^{\text {conv }}=3.91 \times 10^{9}$ & $R_{3}^{\text {conv }}=1.92 \times 10^{9}$ & $R_{4}^{\text {conv }}=2.61 \times 10^{7}$ & $R_{5}^{\text {conv }}=9.26 \times 10^{6}$ \\
\hline
\end{tabular}

${ }^{a}$ By equation (8)

${ }^{\mathrm{b}}$ By equation (10)

${ }^{\mathrm{c}}$ By equation (11)

The probe measures the average temperature of the nonlocalized platinum resistor at the tip. In this model, there is a small portion of resistor in the ballistic air conduction regime ( $T_{l}$ in figure 11). However, given its geometry and the corner effect in the Pt resistor [66], region A is responsible for less than $1 / 1000$ of the total resistance. Therefore, $T_{1}$ can be neglected and only $T_{2}$ is used as the average sensor temperature. Figure 12 shows a plot comparing the probe temperature experimentally obtained by measuring the scanning target of JN device and its temperature calculated using the lumped system model. Uncertainties in the calibration come from various sources, including the uncertainty of JN device temperature measurement, $R_{t s}$ fitting and the TCR of the probe, among these the former two dominate. Uncertainty of the $\mathrm{JN}$ device has been regarded as a constant $( \pm 0.73 \mathrm{~K})$, and $R_{t s}$ provides a maximum $13 \%$ variation in calculated device temperature as interpreted from the measured probe temperature.

In order to quantify the measurement of samples with heated regions smaller than $16 \mu \mathrm{m}$, for example heated nanowires, additional information must be included in the lumped system model. The tip-sample thermal resistance, which will differ with the materials in contact, must be reconsidered and determined [3][27][55]. In addition, the lumped system would require modification to account for the dimensions of the heated region, which dictates the effect of thermal resistance due to air conduction to and from the probe. This can be achieved by varying the quantity of $R_{i}^{\text {air }}$ in the lumped system. The transition to a sub-micrometre heat source varies the predicted temperature measurement by less than $10 \%$ in comparison to the $16 \mu \mathrm{m}$ heaters. One possible method to experimentally determine this variation would be to use the JN device 4-terminal resistor as both a heater and a thermometer, providing a $2 \mu \mathrm{m}$ heated region, comparable to those samples with smaller heated regions. 


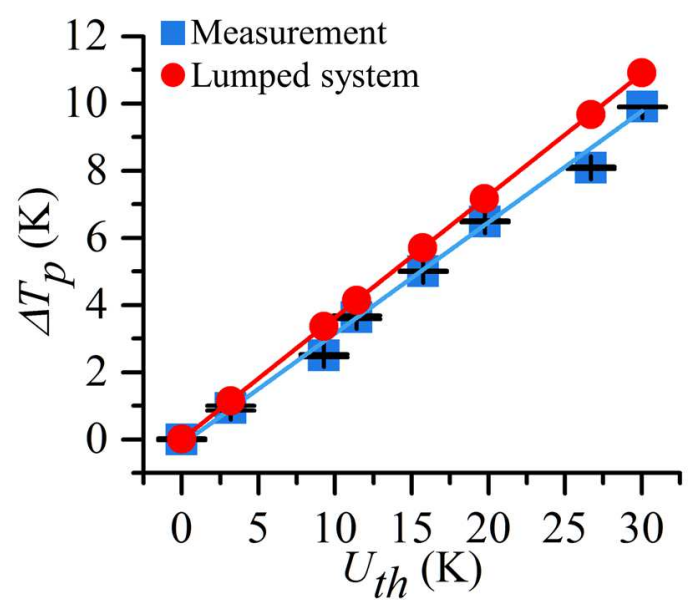

Figure 12. Probe temperature obtaining from experiment and from the lumped system model. $U_{t h}$ is the temperature of the JN device in the experiment, $\Delta T_{p}$ is the probe temperature above the ambient calculated from the output of lock-in amplifier (blue square) in measurement and the $T_{2}$ picked from lumped system (red circle).

Thermal resistance of the whole cantilever can also be extracted from the model, as $2.26 \times 10^{5} \mathrm{~K} / \mathrm{W}$. This value is comparable to that determined by Assy et al. $\left(5.2 \times 10^{5} \mathrm{~K} / \mathrm{W}\right)$, using a very similar self-heated probe in vacuum [3]. The difference can be explained by the effect of the two NiCr protection resistors present on the probes used by Assy but absent in this work. In Assy's work, the power dissipated by the platinum tip resistor was considered to be $2 / 3$ of the power applied to the probe based on the assumption that the platinum tip accounted for $2 / 3$ of the total probe resistance. However, this ratio can vary considerably between probe batches. In addition, the $\mathrm{NiCr}$ resistors would also experience Joule heating, decreasing the thermal gradient along the cantilever, manifesting as a larger cantilever thermal resistance. These limitations are not present in this work.

The thermal resistance of the standard cantilever has been found to be a limitation in detecting materials with thermal conductivity lower than $1 \mathrm{~W} / \mathrm{mK}$ or higher than several hundred [22][67][68]. The lumped system model provides a simple way to evaluate new probe designs, which may be modified to obtain a higher sensitivity to specific sample thermal conductivities. Furthermore, since the thermal interactions between the cantilever and sample are dictated by the area of the cantilever, reducing the effect of air conduction can be achieved by reducing cantilever area. Moreover, the thermal resistance network can be easily converted for use in modelling active probes by substituting the voltage (temperature) source with a current (heating power) source. The relationship between an active probe and sample thermal properties can thus be built to deeply understand heat transfer when measuring complex samples 


\section{Conclusions}

We have designed and fabricated a novel Johnson-noise-based device for the calibration and study of SThM probes. The device provides a known temperature sample with a highly uniform distribution across a suspended membrane, enabling the whole system to be regarded as an isothermal system. A 1D heat transfer equation was built for a SThM probe interacting with the device, which, combined with a FEA model, has been used to characterize the thermal interactions between the probe and sample. This model strongly suggests a larger contact radius than predicted by the frequently used DMT model, indicating a contact with the same radius as the probe's tip radius of curvature. This is the first time this characteristic has been determined in a simple experiment carried out under ambient conditions. Finally, this information was used in the construction of a lumped model considering both the shape and the complex materials of the probe. The model yields a probe thermal resistance in close agreement with that determined by other researchers, and provides a direct and simple way to evaluate design alterations to the probe such as those addressing improved sensitivity as well as enhancing immunity to air conduction artefacts.

By using the SThM under vacuum, the JN noise device could provide accurate temperature calibration. Alternatively, the device can be used under ambient conditions allowing the effect of air conduction to be quantified. The JN device is designed for probes with comparable size as the $\mathrm{SiN}_{\mathrm{x}}$ probe, such as the doped silicon and thermocouple probes. In order to employ the device with other types of probes having larger dimensions (e.g. the Wollaston probe), the device would have to be modified to enlarge the heated regions.

\section{Acknowledgements}

The authors thank the QUANTIHEAT European Project (Grant Agreement No. 604668) for its financial support and the James Watt Nanofabrication Centre (JWNC) for access to facilities. The help of Dr. Donald MacLaren for providing access to the AFM is gratefully acknowledged.

\section{Reference}

[1] Majumdar A 1999 Scanning Thermal Microscopy Annu.Rev. Mater. Sci. 29 505-85

[2] Menges F, Riel H, Stemmer A and Gotsmann B 2012 Quantitative thermometry of nanoscale hot spots Nano Lett. 12 596-601

[3] Assy A and Gomès S 2015 Heat transfer at nanoscale contacts investigated with scanning thermal microscopy Appl. Phys. Lett. 107043105

[4] Simkin M V. and Mahan G D 2000 Minimum Thermal Conductivity of Superlattices Phys. Rev. Lett. 84 927-30

[5] Koh Y K, Cao Y, Cahill D G and Jena D 2009 Heat-transport mechanisms in superlattices Adv. Funct. Mater. 19 610-5 
[6] Persson A I, Koh Y K, Cahill D G, Samuelson L and Linke H 2009 Thermal conductance of InAs nanowire composites Nano Lett. 9 4484-8

[7] Völklein F, Reith H, Cornelius T W, Rauber M and Neumann R 2009 The experimental investigation of thermal conductivity and the Wiedemann-Franz law for single metallic nanowires. Nanotechnology 20 325706

[8] Cretin B, Gomes S, Trannoy N and Vairac P 2006 Scanning Thermal Microscopy Microscale and Nanoscale Heat Transfer Topics in Applied Physics ed S Volz (Berlin/Herdelberg: Springer Berlin Heidelberg) pp 181-238

[9] Mills G, Zhou H, Midha A, Donaldson L and Weaver J M R 1998 Scanning thermal microscopy using batch fabricated thermocouple probes Appl. Phys. Lett. 72 2900-2

[10] Shi L, Zhou J, Kim P, Bachtold A, Majumdar A and McEuen P L 2009 Thermal probing of energy dissipation in current-carrying carbon nanotubes J. Appl. Phys. 105 10-5

[11] Luo K, Shi Z, Varesi J and Majumdar A 1997 Sensor nanofabrication, performance, and conduction mechanisms in scanning thermal microscopy J. Vac. Sci. Technol. B 15349

[12] Gomès S, Assy A and Chapuis P 2015 Scanning thermal microscopy: A review Phys. status solidi 212 477-94

[13] Shi L, Kwon O, Miner A C and Majumdar A 2001 Design and batch fabrication of probes for sub-100 nm scanning thermal microscopy J. Microelectromechanical Syst. 10 370-8

[14] Thiery L, Toullier S, Teyssieux D and Briand D 2008 Thermal Contact Calibration Between a Thermocouple Probe and a Microhotplate J. Heat Transfer 130091601

[15] Dobson P S, Mills G and Weaver J M R 2005 Microfabricated temperature standard based on Johnson noise measurement for the calibration of micro- and nano-thermometers Rev. Sci. Instrum. 76054901

[16] Wielgoszewski G and Gotszalk T 2015 Scanning Thermal Microscopy (SThM): How to Map Temperature and Thermal Properties at the Nanoscale Advances in Imaging and Electron Physics vol 190, ed P W Hawkes (London: Elsevier Inc.) pp 177-221

[17] Li D, Wu Y, Kim P, Shi L, Yang P and Majumdar A 2003 Thermal conductivity of individual silicon nanowires Appl. Phys. Lett. 83 2934-6

[18] Zhou F, Moore A L, Bolinsson J, Persson A, Fröberg L, Pettes M T, Kong H, Rabenberg L, Caroff P, Stewart D A, Mingo N, Dick K A, Samuelson L, Linke H and Shi L 2011 Thermal conductivity of indium arsenide nanowires with wurtzite and zinc blende phases Phys. Rev. B 83 1-10

[19] Chung J, Kim K, Hwang G, Kwon O, Jung S, Lee J W J, Lee J W J and Kim G T 2010 Quantitative temperature measurement of an electrically heated carbon nanotube using the null-point method Rev. Sci. Instrum. 81

[20] Menges F, Riel H, Stemmer A, Dimitrakopoulos C and Gotsmann B 2013 Thermal Transport into Graphene through Nanoscopic Contacts Phys. Rev. Lett. 111205901

[21] Grosse K L, Bae M-H, Lian F, Pop E and King W P 2011 Nanoscale Joule heating, Peltier cooling and current crowding at graphene-metal contacts. Nat. Nanotechnol. $6287-90$

[22] Tovee P, Pumarol M, Zeze D, Kjoller K and Kolosov O 2012 Nanoscale spatial resolution probes for scanning thermal microscopy of solid state materials J. Appl. Phys. 112

[23] Pettes M and Shi L 2013 A Reexamination of Phonon Transport Through a Nanoscale Point Contact in Vacuum J. Heat Transfer 136032401

[24] Hinz M, Marti O, Gotsmann B, Lantz M A and Dürig U 2008 High resolution vacuum scanning thermal microscopy of $\mathrm{HfO} 2$ and $\mathrm{SiO} 2$ Appl. Phys. Lett. 92043122

[25] Shi L and Majumdar A 2002 Thermal Transport Mechanisms at Nanoscale Point Contacts J. Heat Transfer 124329

[26] Nelson B A and King W P 2007 Measuring material softening with nanoscale spatial resolution using heated silicon probes Rev. Sci. Instrum. 78

[27] Lefèvre S, Volz S and Chapuis P O 2006 Nanoscale heat transfer at contact between a hot tip and a substrate Int. J. Heat Mass Transf. 49 251-8 
[28] Kim K, Jeong W, Lee W, Sadat S, Thompson D, Meyhofer E and Reddy P 2014 Quantification of thermal and contact resistances of scanning thermal probes Appl. Phys. Lett. 203107

[29] Menges F, Mensch P, Schmid H, Riel H, Stemmer A and Gotsmann B 2016 Temperature mapping of operating nanoscale devices by scanning probe thermometry Nat. Commun. 710874

[30] Fletcher P C, Lee B and King W P 2011 Thermoelectric voltage at a nanometer-scale heated tip point contact Nanotechnology 23035401

[31] Park K, Cross G L W, Zhang Z M and King W P 2008 Experimental Investigation on the Heat Transfer Between a Heated Microcantilever and a Substrate J. Heat Transfer 130102401

[32] Saha S K and Shi L 2007 Molecular dynamics simulation of thermal transport at a nanometer scale constriction in silicon J. Appl. Phys. 101074304

[33] Derjaguin B V, Muller V M and Toporov Y P 1975 Effect of contact deformations on the adhesion of particles J. Colloid Interface Sci. 53 314-26

[34] Puyoo E, Grauby S, Rampnoux J M, Rouvìre E and Dilhaire S 2010 Thermal exchange radius measurement: Application to nanowire thermal imaging Rev. Sci. Instrum. 81 2-6

[35] Zhang Y, Dobson P S and Weaver J M R 2012 High temperature imaging using a thermally compensated cantilever resistive probe for scanning thermal microscopy J. Vac. Sci. Technol. B 30010601

[36] Gotsmann B, Lantz M A, Knoll A and Dürig U 2009 Nanoscale thermal and mechnical interactions studied using heatable probes Nanotechnology vol 6, ed H Fuchs (Weinheim: Wiley-VCH) pp 121-69

[37] Nelson B and King W 2008 Modeling and Simulation of the Interface Temperature Between a Heated Silicon Tip and a Substrate Nanoscale Microscale Thermophys. Eng. 12 98-115

[38] Gomes S, Trannoy N, Grossel P, Depasse F, Bainier C and Charraut D 2001 D.C. scanning thermal microscopy: Characterisation and interpretation of the measurement Int. J. Therm. Sci. 40 949-58

[39] Lefèvre S and Volz S 2005 3w-scanning thermal microscope Rev. Sci. Instrum. 76033701

[40] Rölke J 1981 Nichrome Thin Film Technology and its Application Electrocompon. Sci. Technol.9 51-7

[41] Zhang Y, Dobson P S and Weaver J M R 2011 Batch fabricated dual cantilever resistive probe for scanning thermal microscopy Microelectron. Eng. 88 2435-8

[42] Cumming D R S, Thoms S, Weaver J M R and Beaumont S P $19963 \mathrm{~nm} \mathrm{NiCr}$ wires made using electron beam lithography and PMMA resist Microelectron. Eng. 30 423-5

[43] Elgaid K, Edgar D L, Ferguson S M, Beaumont S P and Thayne I G 2001 Fabrication of on-wafer MMIC compatible integrated NiCr loads Microelectron. Eng. 57-58 801-6

[44] Kim K J and King W P 2009 Thermal conduction between a heated microcantilever and a surrounding air environment Appl. Therm. Eng. 29 1631-41

[45] Jones W and March N H 1985 Wiedzmann-Franz Law.pdf Theoretical Solid State Physics: NonEquilibrium and Disorder (Mineola, NY: Dover Publications)

[46] Cardenas C, Fabris D, Tokairin S, Madriz F and Yang C Y 2012 Thermoreflectance Measurement of Temperature and Thermal Resistance of Thin Film Gold J. Heat Transfer 134111401

[47] Ftouni H, Blanc C, Tainoff D, Fefferman A D, Defoort M, Lulla K J, Richard J, Collin E and Bourgeois O 2015 Thermal conductivity of silicon nitride membranes is not sensitive to stress Phys. Rev. B 92125439

[48] Dobson P S, Weaver J M R and Mills G 2007 New methods for calibrated Scanning Thermal Microscopy (SThM) Proc. IEEE Sensors 708-11

[49] Bodzenta J, Juszczyk J and Chirtoc M 2013 Quantitative scanning thermal microscopy based on determination of thermal probe dynamic resistance. Rev. Sci. Instrum. 84093702

[50] Ge Y, Zhang Y, Weaver J M R, Zhou H and Dobson P S 2015 Topography-free sample for thermal spatial response measurement of scanning thermal microscopy J. Vac. Sci. Technol. B 33 06FA03

[51] Shi L, Plyasunov S, Bachtold A, McEuen P L and Majumdar A 2000 Scanning thermal microscopy of carbon nanotubes using batch-fabricated probes Appl. Phys. Lett. 774295

[52] Assy A, Lefèvre S, Chapuis P O and Gomès S 2014 Analysis of heat transfer in the water meniscus at the tip-sample contact in scanning thermal microscopy J. Phys. D. Appl. Phys. 47442001 
[53] Kim K, Chung J, Won J, Kwon O, Lee J S, Park S H and Choi Y K 2008 Quantitative scanning thermal microscopy using double scan technique Appl. Phys. Lett. 93203115

[54] Assy A and Gomès S 2015 Temperature-dependent capillary forces at nano-contacts for estimating the heat conduction through a water meniscus Nanotechnology 26355401

[55] Puyoo E, Grauby S, Rampnoux J M, Rouvire E and Dilhaire S 2011 Scanning thermal microscopy of individual silicon nanowires J. Appl. Phys. 109

[56] Zhang X and Grigoropoulos C P 1995 Thermal conductivity and diffusivity of free-standing silicon nitride thin films Rev. Sci. Instrum. 66 1115-20

[57] Bahadur V, Xu J, Liu Y and Fisher T S 2005 Thermal Resistance of Nanowire-Plane Interfaces J. Heat Transfer 127664

[58] Prasher R 2005 Predicting the thermal resistance of nanosized constrictions Nano Lett. 5 2155-9

[59] Chapra S C 2012 Applied Numerical Methods with MATLAB for Engineers and Scientists Applied Numerical Methods with MATLAB for Engineers and Scientists ed M Lange (New York: McGraw-Hill) pp $628-40$

[60] Stoner R J and Maris H J 1993 Kapitza Conductance and Heat-Flow between Solids at Temperatures from 50 to 300K Phys. Rev. B 48 16373-87

[61] Gotsmann B and Lantz M A 2012 Quantized thermal transport across contacts of rough surfaces Nat. Mater. 12 59-65

[62] Gundrum B C, Cahill D G and Averback R S 2005 Thermal conductance of metal-metal interfaces Phys. Rev. B 72245426

[63] Bodzenta J, Chirtoc M and Juszczyk J 2014 Reduced thermal quadrupole heat transport modeling in harmonic and transient regime scanning thermal microscopy using nanofabricated thermal probes $J$. Appl. Phys. 116054501

[64] Puyoo E, Grauby S, Rampnoux J, Claeys W, Rouviere E and Dilhaire S 2010 Simultaneous topographic and thermal imaging of silicon nanowires using a new SThM probe Therm. Investig. ICs Syst. (THERMINIC), 2010 16th Int. Work. 1-6

[65] Holman J P 2010 Heat Transfer (New York: McGraw-Hill)

[66] Baker R J 2008 CMOS Circuit Design, Layout, and Simulation ed S K Tewksbury and J E Brewer (Honoken, New Jersey: John Wiley \& Sons Ltd.)

[67] Bodzenta J, Kaźmierczak-Bałata A, Lorenc M and Juszczyk J 2010 Analysis of Possibilities of Application of Nanofabricated Thermal Probes to Quantitative Thermal Measurements Int. J. Thermophys. 31 150-62

[68] Gomès S, David L, Lysenko V, Descamps A, Nychyporuk T and Raynaud M 2007 Application of scanning thermal microscopy for thermal conductivity measurements on meso-porous silicon thin films $J$. Phys. D. Appl. Phys. 40 6677-83 